\title{
Parker Solar Probe Enters the Magnetically Dominated Solar Corona
}

\author{
J. C. Kasper®
}

BWX Technologies, Inc., Washington, DC 20001, USA

and Climate and Space Sciences and Engineering, University of Michigan, Ann Arbor, Michigan 48109, USA

K. G. Klein® and E. Lichko®

Lunar and Planetary Laboratory, University of Arizona, Tucson, Arizona 85719, USA

Jia Huang(i)

Climate and Space Sciences and Engineering, University of Michigan, Ann Arbor, Michigan 48109, USA

\section{H. K. Chen $\odot$}

Department of Physics and Astronomy, Queen Mary University of London, London E1 4NS, United Kingdom

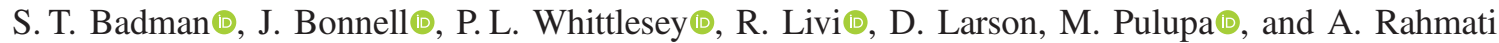
Space Sciences Laboratory at University of California, Berkeley, California, USA

$$
\text { D. Stansby }
$$

Mullard Space Science Laboratory, University College London, Holmbury St. Mary, Surrey RH5 6NT, United Kingdom

K. E. Korreck๑, M. Stevens๑, and A. W. Case $\odot$

Smithsonian Astrophysical Observatory, Cambridge, Massachusetts 02138, USA

\section{S. D. Bale}

Physics Department, University of California, Berkeley, California 94720-7300, USA and Space Sciences Laboratory at University of California, Berkeley, California 94720-7300, USA

M. Maksimovic $\odot$ and M. Moncuquet $\odot$

LESIA, Observatoire de Paris, Universite PSL, CNRS, Sorbonne Universite, Universite de Paris, 5 place Jules Janssen, 92195 Meudon, France

\section{K. Goetz}

School of Physics and Astronomy, University of Minnesota, Minneapolis, Minnesota 55455, USA

\section{J. S. Halekas@}

Department of Physics and Astronomy, University of Iowa, Iowa City, Iowa 52242, USA

D. Malaspina

Laboratory for Atmospheric and Space Physics, University of Colorado Boulder, Boulder, Colorado 80303, USA

Nour E. Raouafi®

The Johns Hopkins Applied Physics Laboratory, Laurel, Maryland 20723, USA

A. Szabo® and R. MacDowall

Heliospheric Physics Laboratory, NASA Goddard Space Flight Center, Greenbelt, Maryland, 20771, USA

Marco Velli®i)

Earth Planetary and Space Sciences, UCLA, California 90095, USA

Thierry Dudok de Wit@

LPC2E, CNRS and University of Orléans, Orléans, France

\section{G. P. Zank}

Department of Space Science and Center for Space Plasma and Aeronomic Research, University of Alabama in Huntsville, Huntsville, Alabama 35805, USA

(Received 31 October 2021; revised 9 November 2021; accepted 15 November 2021;

published 14 December 2021; corrected 15 December 2021) 
The high temperatures and strong magnetic fields of the solar corona form streams of solar wind that expand through the Solar System into interstellar space. At 09:33 UT on 28 April 2021 Parker Solar Probe entered the magnetized atmosphere of the Sun 13 million $\mathrm{km}$ above the photosphere, crossing below the Alfvén critical surface for five hours into plasma in casual contact with the Sun with an Alfvén Mach number of 0.79 and magnetic pressure dominating both ion and electron pressure. The spectrum of turbulence below the Alfvén critical surface is reported. Magnetic mapping suggests the region was a steady flow emerging on rapidly expanding coronal magnetic field lines lying above a pseudostreamer. The sub-Alfvénic nature of the flow may be due to suppressed magnetic reconnection at the base of the pseudostreamer, as evidenced by unusually low densities in this region and the magnetic mapping.

DOI: 10.1103/PhysRevLett.127.255101

Introduction.-The solar corona produces streams of solar wind with sufficient speed to escape the gravitational influence of the Sun and expand through interplanetary space [1]. Near the Sun the pressure and energy density of the corona is magnetically dominated, with magnetic pressure greater than thermal pressure $\left[\beta=n k_{B} T /\left(B^{2} / 2 \mu_{0}\right) \ll 1\right]$ and kinetic energy density, and hydromagnetic Alfvén waves traveling substantially faster than sound waves. This magnetic dominance is an essential aspect of the physics responsible for coronal heating, for shaping coronal structures such as loops and streamers, and for accelerating solar wind. It is also a key differentiator between coronal plasma still connected to the Sun and the free-streaming solar wind that has been directly probed by spacecraft to date.

Neglecting corrections for pressure anisotropy and multiple species, the speed of shear hydromagnetic Alfvén waves traveling along magnetic fields is determined by the local magnetic field magnitude and the density of the fluid $v_{A}=B / \sqrt{\mu_{0} \rho}$ [2]. The Alfvén critical surface marks the distance $r_{A}$ where the radial component of the solar wind speed $v_{\mathrm{r}}$ surpasses $v_{A}$ and the kinetic energy density of the solar wind is less than the magnetic energy density. Below $r_{A}$, the solar wind is Alfvénically causally connected to the Sun and magnetic forces dictate the behavior of the charged particles. Torques from the rotating Sun reach a maximum at $r_{A}$ and set the angular momentum loss and spin-down rate of the Sun $[3,4]$. The mass flux of the solar wind is also governed by the plasma properties within $r_{A}$ [5]. The sub-Alfvénic region is also where a number of proposed mechanisms to heat the corona and accelerate the solar wind have been suggested to operate, including the dissipation of magnetic turbulence [6-11] or resonant damping of ion-cyclotron waves [12,13], leading to the preferential heating of minor ions $[14,15]$. The solar wind below this boundary has been proposed as the source of magnetic field reversals, or so-called switchbacks, first

Published by the American Physical Society under the terms of the Creative Commons Attribution 4.0 International license. Further distribution of this work must maintain attribution to the author(s) and the published article's title, journal citation, and DOI. discovered by Ulysses [16] and seen in large numbers by Parker Solar Probe (PSP) [17-21]. Switchbacks are nonlinear Alfvén waves $[22,23]$ that may be associated with the global circulation of open magnetic flux at the base of the corona $[11,18]$ or the transition between structured flow and the initiation of the turbulent cascade observed throughout the inner heliosphere [24-26].

To date, measurements of sub-Alfvénic solar wind have been limited to conditions with thermal pressure similar to or greater than magnetic pressure, and to situations arising from the interaction of solar wind streams far from the Sun $[27,28]$, or to intervals sufficiently short in duration or physical size to prevent the conclusion that they represent steady streams of sub-Alfvénic solar wind [29]. In this Letter we show the first observations of a sub-Alvénic solar wind stream by PSP in the solar wind acceleration region with a median Alfvén Mach number of $M_{A}=0.79$ and magnetic energy dominating both kinetic and thermal energy. The interval lasted nearly $5 \mathrm{~h}$, during which time the spacecraft moved from 19.7 to 18.4 solar radii $\left(R_{\odot} \equiv 6.95 \times 10^{5} \mathrm{~km}\right)$ from the center of the Sun and traversed seven degrees in solar longitude. The duration of the stream is more than an order of magnitude larger than that associated with the longest wavelength turbulent fluctuations seen by PSP, demonstrating this is not a transient phenomenon driven by local conditions. The stream appears to have been produced across the boundary of a change in the solar wind source from a midlatitude to a polar coronal hole.

Data.-The data presented in this Letter were collected over the course of the eighth encounter of the Sun by Parker Solar Probe in April 2021 (E8) and have been released for public use through the NASA PSP archive. Thermal plasma properties are measured by the PSP solar wind electron, alphas, and protons (SWEAP) instrument suite [30], including the Solar Probe Cup (SPC) [31], solar probe analyzers (SPAN) electron [32], and SPAN ion plasma data. The radial component of the solar wind proton velocity used in Fig. 1 was determined by calculating the moments of proton velocity measured by SPC. Both SWEAP ion instruments (SPC and SPAN ion) measure radial velocities that agree to within a few $\mathrm{km} \mathrm{s}^{-1}$ during this time period. Uncertainties in 


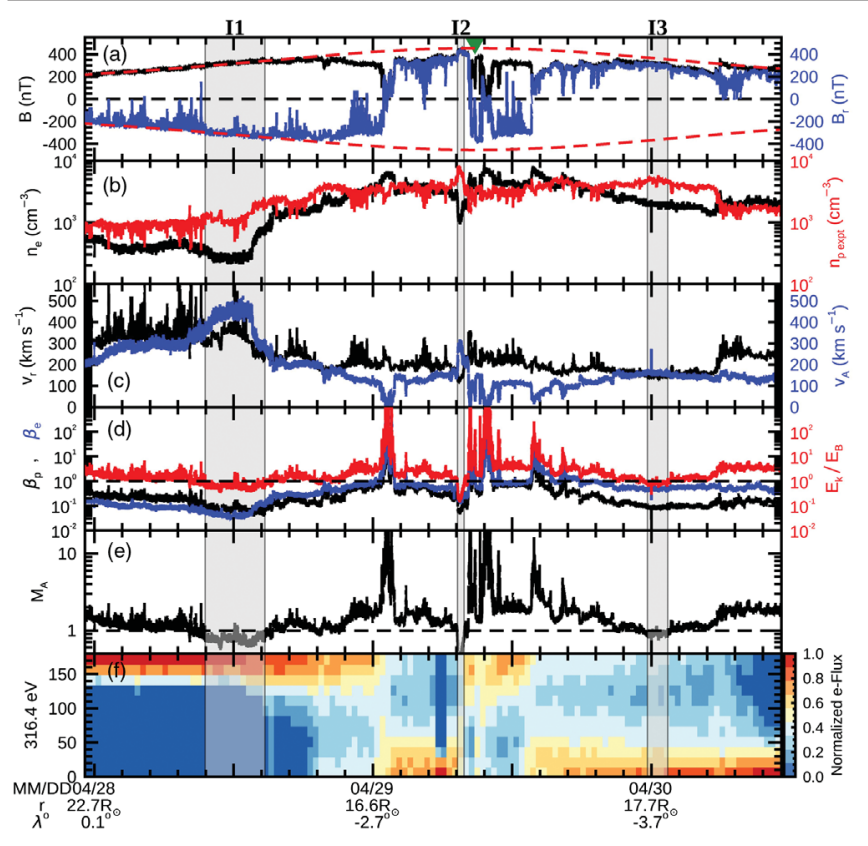

FIG. 1. An overview of the eighth solar encounter (E8) by PSP. (a) Total amplitude $\left(B\right.$, black) and radial component $\left(B_{r}\right.$, blue $)$ of magnetic field, enveloped by the scaled $B$ prediction (red). The green triangle indicates a perihelion at $15.9 R_{\odot}$ on 29 April 2021 at 08:48 UT. (b) Electron density ( $n_{e}$, black) and the expected proton density ( $n_{p \text { expt }}$ based on speed and distance, red). (c) Proton radial speed $\left(v_{r}\right.$, black) and the Alfvén speed $\left(v_{A}\right.$, blue). (d) Proton $\left(\beta_{p}\right.$, black) and electron $\left(\beta_{e}\right.$, blue) plasma beta together with the kinetic to magnetic energy density ratio $\left(E_{k} / E_{B}\right.$, red). (e) Radial Alfvén Mach number $\left(M_{A}\right)$. (f) Normalized electron pitch angle distribution at $316.4 \mathrm{eV}$. The date (month/ day), distance $r$, and latitude $\lambda^{\circ}$ relative to the solar equator are indicated at daily intervals. PSP was in sub-Alfvénic flow for three periods in this encounter as shown by the shaded regions.

the measurements are described in the instrument papers and elsewhere [33]. Magnetic field data from the outboard FIELDS magnetometer and a determination of the electron density $n_{e}$ from the peak in the spectrum of quasithermal noise fluctuations was also used [34-36]. Power spectral density measurements are limited by magnetic field instrument noise above $10 \mathrm{~Hz}$ in this period. Mass density for the Alfvén speed is estimated by multiplying $n_{e}$ by $m_{p}$. Observations of electrons with a center energy of $316.4 \mathrm{eV}$ and width of $22 \mathrm{eV}$ by the two SPAN electron sensors were combined, along with the FIELDS determination of the magnetic field direction, to create electron pitch angle distributions and track the direction of the electron heat flux along the local magnetic field. Since the electron heat flux always flows away from the corona into space, simultaneous changes in the polarity of the radial component of the magnetic field and the direction of the electron heat flux are used to determine when PSP has crossed the heliospheric current sheet (HCS) that divides solar wind connected to inward or outward magnetic field polarity $[33,37]$.

Observations of sub-Alfvénic solar wind.-PSP crossed below the Alfvén critical surface within three intervals over the 2.5 day period near the E8 perihelion shown in Fig. 1. The three intervals (I1-I3), each of which lasted more than $30 \mathrm{~min}$, are indicated in the figure by shading and described in Table I.

Both $B$ and $B_{r}$ increased toward perihelion as expected, roughly following the envelope expected from an inverse square dependence on distance [38]. Three major crossings of the HCS are indicated by the drop in $B$, reversal in polarity of $B_{r}$, increase in $\beta$, and reversal of the sign of the electron heat flux. Large numbers of traveling magnetic switchbacks or nonlinear Alfvén waves, evidenced by the short period rotations in the direction of $B_{r}$ with no change in magnitude, are seen throughout the encounter but occur at a notably lower rate when $P S P$ is below the critical surface. The magnitudes of $B$ and $B_{r}$ more closely adhere to the predicted envelope when PSP is below the Alfvén critical surface.

The observed electron density $n_{e}$ is also compared to an expected value $n_{p \text { expt }} v_{r} R_{\mathrm{sc}}^{2}=10^{4.85} v_{r}^{-0.54}(1 \text { A.U. })^{2}$ derived from Wind spacecraft observations near Earth and scaled for distance from the Sun. The radial trends in $v_{r}$ and $v_{A}$ make it more likely that PSP would cross below the Alfvén critical point as the spacecraft moved closer to the Sun. The main factor contributing to these three specific intervals being the first is the unusually low density seen in all three cases, about 2-5 times lower than would be expected from scaling the typical values.

I1 is the primary focus of this Letter because $M_{A}$ was consistently below unity for more than $300 \mathrm{~min}$, the interval was far from the HCS structure, and because for the first time the thermal and kinetic energy density of both ions and electrons was measurably less than the magnetic energy density. Thus I1 marks the first time a spacecraft has passed into the magnetically dominated solar corona. 12 shows a significant dip of $M_{A}$ to even lower values, but this interval

TABLE I. Start and end time, corresponding distance from the Sun and Carrington longitude of $P S P$, and median $M_{A}$ within the three intervals below the Alfvén critical surface.

\begin{tabular}{|c|c|c|c|c|c|c|c|}
\hline Interval & Start (UT) & End (UT) & Start $\left(R_{\odot}\right)$ & End $\left(R_{\odot}\right)$ & Start (deg) & End (deg) & Median $M_{A}$ \\
\hline I1 & 2021-04-28 09:33 & 2021-04-28 $14: 42$ & 19.8 & 18.4 & 42.2 & 49.0 & 0.79 \\
\hline I2 & 2021-04-29 07:18 & 2021-04-29 07:52 & 16.0 & 16.0 & 80.1 & 81.4 & 0.49 \\
\hline I3 & 2021-04-29 23:40 & 2021-04-30 01:24 & 17.7 & 18.0 & 112.7 & 115.5 & 0.88 \\
\hline
\end{tabular}




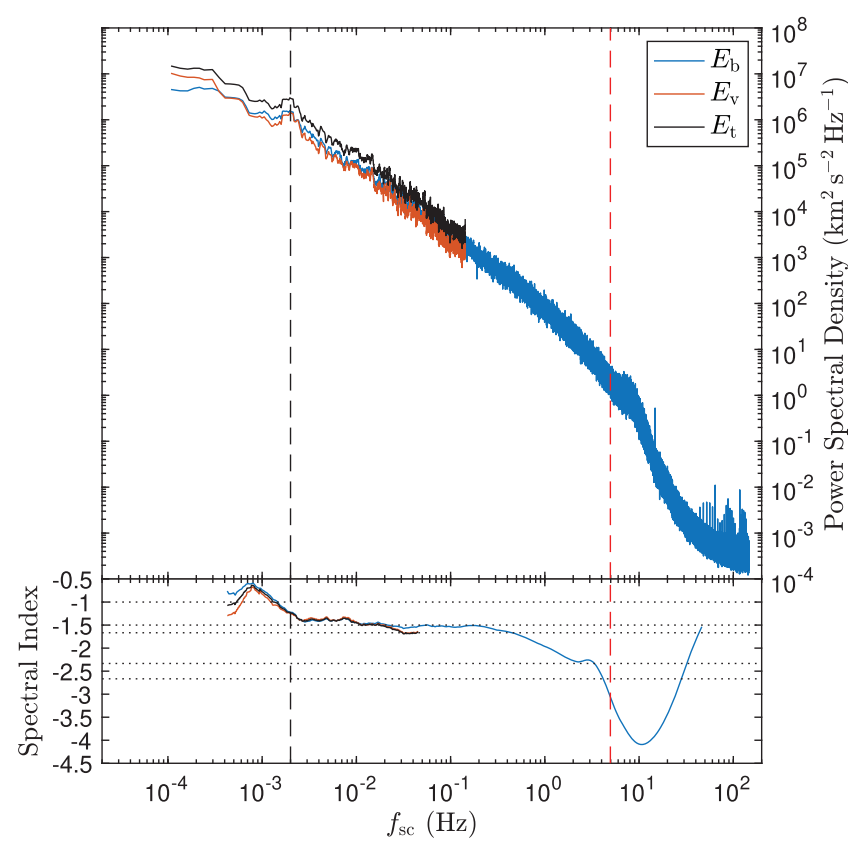

FIG. 2. Power spectrum (upper panel) and spectral index (lower panel) of magnetic $\left(E_{b}\right)$, velocity $\left(E_{v}\right)$, and total energy $\left(E_{t}\right)$ fluctuations in a $5 \mathrm{~h}$ period within $\mathrm{I} 1$. The break between the lowfrequency " $1 / f$ " range and the turbulent inertial range is marked with vertical dashed lines at $f_{\mathrm{sc}} \sim 2 \times 10^{-3} \mathrm{~Hz}$ (black) and the ion cyclotron period (red). The horizontal dotted lines correspond to -1 for the $1 / f$ range and $-3 / 2$ and $-5 / 3$ as model predictions for the turbulence inertial range [41].

is relatively short and could be a transient associated with the nearby HCS. I3 lasts for about $100 \mathrm{~min}$, but $M_{A}$ does not drop significantly below unity.

$M_{A}$ is reliably below unity in I1 even when adjusting the measurements by more than their known uncertainties or by including higher order corrections to $v_{r}$ and $v_{A}$ arising from center of mass, pressure anisotropy, or minor ion contributions including artificially raising the percentage of helium in the solar wind to $10 \%$ by number density, twice the highest value seen in steady solar wind $[39,40]$.

Figure 2 shows the trace power spectral density of fluctuations in the magnetic field, velocity, and total energy as a function of spacecraft-frame frequency $f_{\text {sc }}$ for the $5 \mathrm{~h}$ period within I1. Normalized fast Fourier transforms of the time series of $\mathbf{v}$ and $\mathbf{b}=\mathbf{B} / \sqrt{\mu_{0} n_{e 0} m_{p}}$ were performed to calculate the power spectral densities and the components of each summed to give the trace power spectral densities $E_{b}$ and $E_{v}$. These spectra were then smoothed with a running average in frequency space of 10 data points. The total energy spectrum was calculated as $E_{t}=E_{b}+E_{v}$. The spectrum for $E_{b}$ extends to higher frequencies due to the faster sampling of the magnetometer, but the flattening in the spectrum above $10 \mathrm{~Hz}$ is due to the noise floor of the instrument and not the plasma. The spectral index, in the lower panel of Fig. 2, for each quantity was found by taking a sliding window of one decade in spacecraft-frame frequency, $f_{\mathrm{sc}}$, over the smoothed spectra and calculating the best-fit linear gradient in log-log space over this window. The spectral break between the energy-containing $1 / f$ range (which is shallower than $f_{\mathrm{sc}}^{-1}$ here) and the $f_{\mathrm{sc}}^{-3 / 2}$ inertial range is at $f_{\mathrm{sc}} \sim 2 \times 10^{-3} \mathrm{~Hz}$ and is indicated with the black dashed line. This break is associated with the outer scale, or largest scale fluctuation that can be attributed to turbulence [42]. The $1 / f$ range and break is clearer when using $15 \mathrm{~h}$ of observations including I1 [43]. The $\sim 500 \mathrm{~s}$ time period corresponding to the measured outer scale is 37 times shorter than the duration of I1, meaning that the sub-Alfvénic nature of I1 cannot be due to the passage of a large turbulent eddy. Instead I1 is a large-scale stream structure, most likely relating to a sub-Alfvénic flow all the way from the Sun to the spacecraft. There is a small enhancement in power at the break scale. At higher frequencies there is still a clear $-3 / 2$ inertial range magnetic field spectrum, although it is shallower at low frequencies. A small enhancement is seen at ion cyclotron scales, and then the spectrum drops steeply before reaching the instrument noise floor.

Solar surface source.-To trace the flows in the three intervals back to their sources deep in the atmosphere of the Sun, three-dimensional reconstruction of the magnetic field between the photosphere of the Sun and PSP is derived using the potential field source surface (PFSS) model [44-47]. The PFSS model can uniquely specify the coronal field once two surface boundary conditions are specified: a map of the observed photospheric magnetic field assembled from magnetograms and a stipulation that all remaining magnetic fields are radial at an outer source surface. For this Letter a PFSS solution was developed using an ADAPT-GONG magnetogram recorded on 1 May 2021 shortly after PSP reached perihelion (29 April 2021) and a source surface height of $2.0 R_{\odot}$ selected to accurately reproduce low latitude coronal holes observed in STEREO spacecraft extreme ultraviolet images of the Sun during the encounter.

The output of the PFSS model is depicted in Fig. 3. The E8 trajectory of PSP over the solar surface is shown in blue and red running across the middle of the plot and forms a loop where superrotation with respect to the solar surface occurs. The color indicates the in situ measured magnetic polarity by FIELDS, where red indicates a positive, antisunward vector and blue indicates a negative, sunward-directed field. The polarity inversion line (solid black curve) is seen to cut the PSP trajectory at the locations where the in situ data changes polarity indicating consistency. Curves connecting the PSP trajectory to the solar surface indicate the modeled coronal field line connection. The color of these field lines utilizes the Alfvén Mach number shown in Fig. 1: Green colors indicate standard super-Alfvénic intervals, and white and purple colors indicate sub-Alfvénic intervals. A white portion on the PSP trajectory indicates the location where 

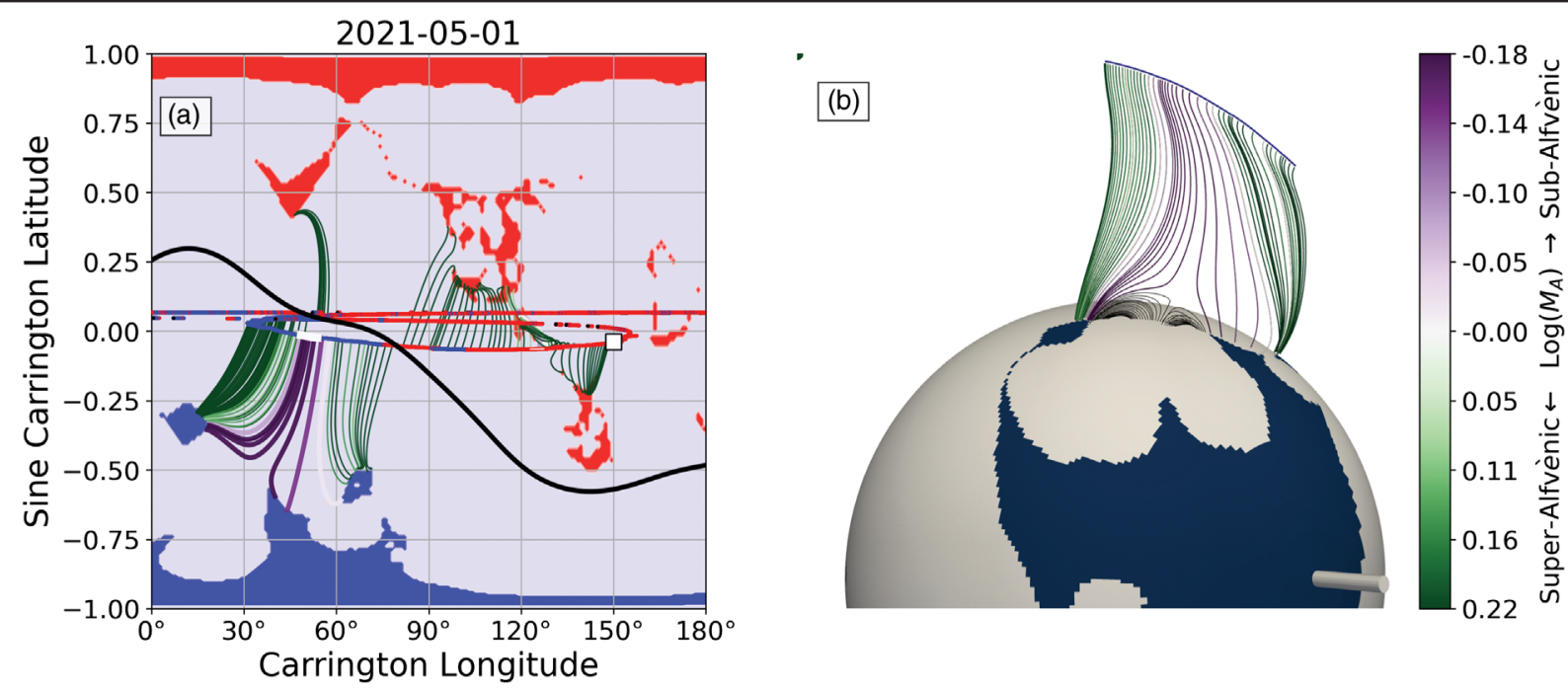

FIG. 3. Mapping of solar wind from PSP to the solar surface using a PFSS magnetic field model. Green and purple lines show connection from PSP to photosphere and color of lines shows Alfvén Mach number, with thick purple and white being the sub-Alfvénic periods. (a) 2D Carrington projection with the heliospheric current sheet (black line), model coronal holes (red or blue regions), and PSP's trajectory projected down to $2.0 R_{\odot}$ (blue or red line) with a square showing location at the start of 1 May 2021 . (b) 3D PSP connected field lines in green or purple, and underlying pseudostreamer field lines in black. $P S P$ traverses from left to right in this plot. $P S P$ crossed about $7^{\circ}$ in longitude in the first interval while the photospheric sources map to two small regions about $40^{\circ}$ apart on the Sun. The modeled angular distance from the HCS was approximately $2^{\circ}-4^{\circ}$ at this time.

PSP measured sub-Alfvénic solar wind speeds. A large white square indicates PSP's location on the date of the model run shown. The ballistic mapping utilizes SWEAP/ SPAN-ion-measured solar wind velocity to estimate the shift in longitude between the outer corona and PSP. While this method makes the crude assumption of no solar wind acceleration or transverse solar wind flow on its path out from the corona, the uncertainty is greatly reduced (i) by the fact that these two effects counteract each other [48,49], and (ii) by PSP's low heliocentric distances which mean that a solar wind variation as large as $200 \mathrm{~km} / \mathrm{s}$ would correspond to a mapping error of less than $5^{\circ}$ on the source surface.

As PSP passed through sub-Alfvénic interval I1, the connection started on the edge of an isolated negative polarity equatorial coronal hole and transitioned to the boundary of a southern polar coronal hole extension, likely crossing a pseudostreamer or quasiseparatrix structure. Pseudostreamers and quasiseperatrix structures are magnetic topologies where a small motion of the spacecraft is associated with a large jump in the location of the magnetic footpoint of the solar wind source with no change in polarity, and have been proposed as playing a critical role in the structure and acceleration of solar wind [50]. In the case of I1, PSP moved through about $7^{\circ}$ in longitude while the footpoint source moved by nearly $40^{\circ}$ over the surface of the Sun. This mapping result for I1 was found to be robust, occurring with magnetograms taken up to 2 weeks on either side of perihelion including times when the coronal hole was visible from Earth [43]. This latter point is important since the actual connection occurred while PSP's footpoint was behind the solar East limb. The result also persists if we assume corotational effects dominate over solar wind acceleration such that the ballistic mapping is replaced with direct radial field lines between PSP and the PFSS domain; in this case the mapping is weighted more towards the equatorial coronal hole, but still associated with the same pseudostreamer structure. Additionally, extreme ultraviolet (EUV) radiation observations at these later times (5 May 2021 onwards) show there was solar activity nearly colocated with the coronal hole, with bright closed loops apparent just south of the EUV-dark coronal hole.

I3 also involves a connection to a low latitude equatorial coronal hole (of positive polarity), although it is much shorter lived and the specific source was less robust to magnetogram evolution, potentially being affected by transient eruptions. This event occurred while the source was visible from Earth. Reproducing coronal holes in this region seen in EUV was the main driver to the choice of source surface height $\left(2.0 R_{\odot}\right)$; higher source surface heights did not capture the southern hemisphere positive coronal hole around $140^{\circ}$ longitude.

Comparison to recent predictions. - The fact that PSP crossed below the Alfvén critical surface for the first time in 2021 and at distances of $16-20 R_{\odot}$ from the Sun is consistent with earlier predictions $[15,51,52]$. One recent study combined solar wind measurements by the Wind spacecraft with a simple radial scaling for each quantity to calculate the typical height of the Alfvén critical surface as a function of time, and predicted that PSP would cross below the surface in 2021 due to increasing solar activity and the continued lowering of the PSP perihelion through encounters with Venus [15]. Figure 4 updates the 


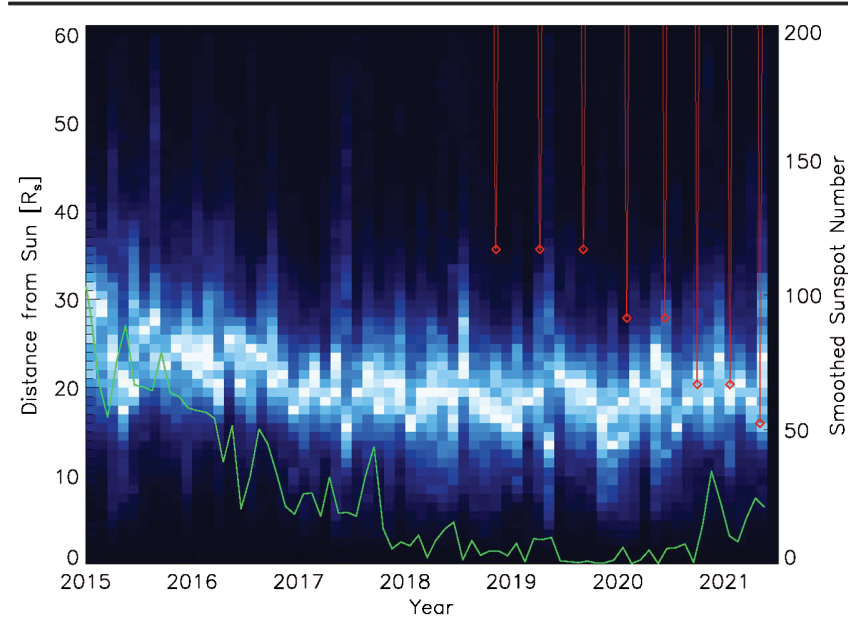

FIG. 4. Comparison of PSP trajectory (red) and prediction of location of the Alfvén critical surface (blue background) using Wind observations at 1 A.U. (updated from [15] with same power law scalings for radial variations in density and magnetic field) and smoothed sunspot number (green). PSP crossing into subAlfvénic flow is consistent with 1 au observations from 2021 extrapolated inwards. Probability and duration of sub-Alfvénic encounters should increase as Alfvén surface expands outwards due to increasing solar activity and PSP perihelion is lowered through additional encounters with Venus.

calculations from that study and includes the PSP trajectory, and the lower perihelion of E8 is coincident with an increase in the predicted surface height. This crossing was also consistent with predictions based on radial extrapolation from earlier encounters, e.g. [52].

Discussion.-A $5 \mathrm{~h}$ interval on 28 April 2021 is the first time we were aware of a spacecraft entering the magnetically dominated atmosphere of the Sun, with a mean $M_{A} \sim 0.78$ and magnetic energy larger than both the kinetic and thermal particle energy. The power spectral density of fluctuations in the magnetic field, velocity, and total energy was measured within the first interval below the Alfvén critical surface. Compared to prior observations in the super-Alfvénic solar wind where the $1 / f$ range was less well defined, there was a potential enhancement in power at the break between the $1 / f$ and inertial range, and the slope of the spectrum of turbulence within the lower frequency side of the inertial range was more shallow than $3 / 2$. Enhanced power at the break scale has been predicted as a signature of parametric instability and inverse cascade and may be an indicator of novel heating and dissipation physics [53].

Magnetic field mapping robustly suggests that the source of this first sub-Alfvénic interval is solar wind emerging from an expanding magnetic field above a pseudostreamer, and demonstrates a link between pseudostreamers or quasiseperatrix layers and solar wind formation. The drop in $M_{A}$ in this interval is predominantly due to the atypically low plasma density and not a change in $B$. There are far fewer switchbacks in this sub-Alfvénic interval than in the surrounding super-Alfvénic plasma. Given the magnetic mapping and the low density this could be an an indication that switchbacks are correlated with the presence of magnetic reconnection on the surface of the Sun, and that lower reconnection rates led to less mass being injected into this solar wind stream [20]. However, the reduction is also constant with predictions that switchbacks are formed above the Alfvén critical point [21]. Additional observations below the Alfvén critical surface at different Mach numbers and with solar sources will reveal the location and process of switchback formation.

These first observations of the magnetically dominated solar corona by Parker Solar Probe demonstrate the unique insights the mission is able to provide into the physics of coronal heating and solar wind formation through direct observation. We can anticipate further discoveries as the spacecraft descends to its final perihelion of $9.86 R_{\odot}$ and spends increasing time within the magnetic atmosphere of our Sun.

The supporting data for this Letter are openly available from the NASA Space Physics Data Facility [54-61]. Additional SWEAP data and information are available at the SWEAP web page [62].

The SWEAP investigation and this study are supported by the PSP mission under NASA Contract No. NNN06AA01C. C. H. K. C. was supported by STFC Ernest Rutherford Fellowship No. ST/N003748/2 and STFC Consolidated Grant No. ST/T00018X/1. D. S. was supported by STFC Consolidated Grant No. ST/S000240/ 1. SWEAP and FIELDS measurements were analyzed and graphics developed in the Interactive Data Language (IDL). The PFSS models were generated using the PFSSPY Python package [63]. This work utilizes data obtained by the Global Oscillation Network Group (GONG) program.

[1] E. N. Parker, Astrophys. J. 128, 664 (1958).

[2] H. Alfvén, Nature (London) 150, 405 (1942).

[3] E. J. Weber and L. Davis Jr., Astrophys. J. 148, 217 (1967).

[4] E. Marsch and A. K. Richter, J. Geophys. Res. 89, 5386 (1984).

[5] E. Leer and T. E. Holzer, J. Geophys. Res. 85, 4681 (1980).

[6] M. Heinemann and S. Olbert, J. Geophys. Res. 85, 1311 (1980).

[7] M. Velli, Astron. Astrophys. 270, 304 (1993), https://ui .adsabs.harvard.edu/abs/1993A\%26A...270..304V/abstract.

[8] W. H. Matthaeus, G. P. Zank, S. Oughton, D. J. Mullan, and P. Dmitruk, Astrophys. J. Lett. 523, L93 (1999).

[9] S. R. Cranmer, A. A. van Ballegooijen, and R. J. Edgar, Astrophys. J. Suppl. Ser. 171, 520 (2007).

[10] B. D. G. Chandran, J. Plasma Phys. 87, 905870304 (2021).

[11] G. P. Zank, L.-L. Zhao, L. Adhikari, D. Telloni, J. C. Kasper, and S. D. Bale, Phys. Plasmas 28, 080501 (2021).

[12] S. R. Cranmer, Astrophys. J. 532, 1197 (2000). 
[13] J. V. Hollweg and P. A. Isenberg, J. Geophys. Res. 107, 1147 (2002).

[14] J. L. Kohl, G. Noci, E. Antonucci, G. Tondello, M. C. E. Huber, S. R. Cranmer, L. Strachan, A. V. Panasyuk, L. D. Gardner, M. Romoli, S. Fineschi, D. Dobrzycka, J. C. Raymond, P. Nicolosi, O. H. W. Siegmund, D. Spadaro, C. Benna, A. Ciaravella, S. Giordano, S. R. Habbal, M. Karovska, X. Li, R. Martin, J. G. Michels, A. Modigliani, G. Naletto, R. H. O’Neal, C. Pernechele, G. Poletto, P. L. Smith, and R. M. Suleiman, Astrophys. J. Lett. 501, L127 (1998).

[15] J. C. Kasper and K. G. Klein, Astrophys. J. Lett. 877, L35 (2019).

[16] A. Balogh, R. J. Forsyth, E. A. Lucek, T. S. Horbury, and E. J. Smith, Geophys. Res. Lett. 26, 631 (1999).

[17] T. S. Horbury, T. Woolley, R. Laker, L. Matteini, J. Eastwood, S. D. Bale, M. Velli, B. D. G. Chandran, T. Phan, N. E. Raouafi, K. Goetz, P. R. Harvey, M. Pulupa, K. G. Klein, T. Dudok de Wit, J. C. Kasper, K. E. Korreck, A. W. Case, M. L. Stevens, P. Whittlesey, D. Larson, R. J. MacDowall, D. M. Malaspina, and R. Livi, Astrophys. J. Suppl. Ser. 246, 45 (2020).

[18] L. A. Fisk and J. C. Kasper, Astrophys. J. Lett. 894, L4 (2020).

[19] G. P. Zank, M. Nakanotani, L. L. Zhao, L. Adhikari, and J. Kasper, Astrophys. J. 903, 1 (2020).

[20] S. D. Bale, T. S. Horbury, M. Velli, M. I. Desai, J. S. Halekas, M. D. McManus, O. Panasenco, S. T. Badman, T. A. Bowen, B. D. G. Chandran, J. F. Drake, J. C. Kasper, R. Laker, A. Mallet, L. Matteini, T. D. Phan, N. E. Raouafi, J. Squire, L. D. Woodham, and T. Wooley, arXiv:2109 .01069 .

[21] N. A. Schwadron and D. J. McComas, Astrophys. J. 909, 95 (2021).

[22] J. W. Belcher and L. Davis Jr., J. Geophys. Res. 76, 3534 (1971).

[23] B. T. Tsurutani, G. S. Lakhina, A. Sen, P. Hellinger, K.-H. Glassmeier, and A. J. Mannucci, J. Geophys. Res. Space Phys. 123, 2458 (2018).

[24] C. E. DeForest, W. H. Matthaeus, N. M. Viall, and S. R. Cranmer, Astrophys. J. 828, 66 (2016).

[25] D. Ruffolo, W. H. Matthaeus, R. Chhiber, A. V. Usmanov, Y. Yang, R. Bandyopadhyay, T. N. Parashar, M. L. Goldstein, C. E. DeForest, M. Wan, A. Chasapis, B. A. Maruca, M. Velli, and J. C. Kasper, Astrophys. J. 902, 94 (2020).

[26] J. Squire, B. D. G. Chandran, and R. Meyrand, Astrophys. J. Lett. 891, L2 (2020).

[27] A. V. Usmanov, M. L. Goldstein, K. W. Ogilvie, W. M. Farrell, and G. R. Lawrence, J. Geophys. Res. Space Phys. 110, A01106 (2005).

[28] C. W. Smith, D. J. Mullan, N. F. Ness, R. M. Skoug, and J. Steinberg, J. Geophys. Res. Space Phys. 106, 18625 (2001).

[29] D. Stansby, Research Notes of the AAS 4, 51 (2020).

[30] J. C. Kasper, R. Abiad, G. Austin, M. Balat-Pichelin, S. D. Bale, J. W. Belcher, P. Berg, H. Bergner, M. Berthomier, and J. Bookbinder, Space Sci. Rev. 204, 131 (2016).

[31] A. W. Case, J. C. Kasper, M. L. Stevens, K. E. Korreck, K. Paulson, P. Daigneau, D. Caldwell, M. Freeman, T. Henry, B. Klingensmith, J. A. Bookbinder, M. Robinson, P. Berg,
C. Tiu, J. Wright, K. H., M. J. Reinhart, D. Curtis, M. Ludlam, D. Larson, P. Whittlesey, R. Livi, K. G. Klein, and M. M. Martinović, Astrophys. J. Suppl. Ser. 246, 43 (2020).

[32] P. L. Whittlesey, D. E. Larson, J. C. Kasper, J. Halekas, M. Abatcha, R. Abiad, M. Berthomier, A. W. Case, J. Chen, D. W. Curtis, G. Dalton, K. G. Klein, K. E. Korreck, R. Livi, M. Ludlam, M. Marckwordt, A. Rahmati, M. Robinson, A. Slagle, M. L. Stevens, C. Tiu, and J. L. Verniero, Astrophys. J. Suppl. Ser. 246, 74 (2020).

[33] J. C. Kasper, S. D. Bale, J. W. Belcher, M. Berthomier, A. W. Case, B. D. G. Chandran, D. W. Curtis, D. Gallagher, S. P. Gary, L. Golub, J. S. Halekas, G. C. Ho, T. S. Horbury, Q. Hu, J. Huang, K. G. Klein, K. E. Korreck, D. E. Larson, R. Livi, B. Maruca, B. Lavraud, P. Louarn, M. Maksimovic, M. Martinovic, D. McGinnis, N. V. Pogorelov, J. D. Richardson, R. M. Skoug, J. T. Steinberg, M. L. Stevens, A. Szabo, M. Velli, P. L. Whittlesey, K. H. Wright, G. P. Zank, R. J. MacDowall, D. J. McComas, R. L. McNutt, M. Pulupa, N. E. Raouafi, and N. A. Schwadron, Nature (London) 576, 228 (2019).

[34] S. D. Bale, K. Goetz, P. R. Harvey, P. Turin, J. W. Bonnell, T. Dudok de Wit, R. E. Ergun, R. J. MacDowall, M. Pulupa, and M. Andre, Space Sci. Rev. 204, 49 (2016).

[35] S. D. Bale, S. T. Badman, J. W. Bonnell, T. A. Bowen, D. Burgess, A. W. Case, C. A. Cattell, B. D. G. Chandran, C. C. Chaston, C. H. K. Chen, J. F. Drake, T. D. de Wit, J.P. Eastwood, R. E. Ergun, W. M. Farrell, C. Fong, K. Goetz, M. Goldstein, K. A. Goodrich, P. R. Harvey, T. S. Horbury, G. G. Howes, J. C. Kasper, P. J. Kellogg, J. A. Klimchuk, K. E. Korreck, V. V. Krasnoselskikh, S. Krucker, R. Laker, D. E. Larson, R. J. MacDowall, M. Maksimovic, D. M. Malaspina, J. Martinez-Oliveros, D. J. McComas, N. Meyer-Vernet, M. Moncuquet, F. S. Mozer, T. D. Phan, M. Pulupa, N. E. Raouafi, C. Salem, D. Stansby, M. Stevens, A. Szabo, M. Velli, T. Woolley, and J. R. Wygant, Nature (London) 576, 237 (2019).

[36] M. Moncuquet, N. Meyer-Vernet, K. Issautier, M. Pulupa, J. W. Bonnell, S. D. Bale, T. Dudok de Wit, K. Goetz, L. Griton, P. R. Harvey, R. J. MacDowall, M. Maksimovic, and D. M. Malaspina, Astrophys. J. Suppl. Ser. 246, 44 (2020).

[37] E. J. Smith, B. T. Tsurutani, and R. L. Rosenberg, J. Geophys. Res. Space Phys. 83, 717 (1978).

[38] S. T. Badman, S. D. Bale, A. P. Rouillard, T. A. Bowen, J. W. Bonnell, K. Goetz, P. R. Harvey, R. J. MacDowall, D. M. Malaspina, and M. Pulupa, Astron. Astrophys. 650, A18 (2021).

[39] B. L. Alterman, J. C. Kasper, M. L. Stevens, and A. Koval, Astrophys. J. 864, 112 (2018).

[40] J. C. Kasper, M. L. Stevens, A. J. Lazarus, J. T. Steinberg, and K. W. Ogilvie, Astrophys. J. 660, 901 (2007).

[41] C. H. K. Chen, J. Plasma Phys. 82, 535820602 (2016).

[42] C. H. K. Chen, S. D. Bale, J. W. Bonnell, D. Borovikov, T. A. Bowen, D. Burgess, A. W. Case, B. D. G. Chandran, T. D. de Wit, K. Goetz, P. R. Harvey, J. C. Kasper, K. G. Klein, K. E. Korreck, D. Larson, R. Livi, R. J. MacDowall, D. M. Malaspina, A. Mallet, M. D. McManus, M. Moncuquet, M. Pulupa, M. L. Stevens, and P. Whittlesey, Astrophys. J. Suppl. Ser. 246, 53 (2020). 
[43] See Supplemental Material at http://link.aps.org/ supplemental/10.1103/PhysRevLett.127.255101 for supplemental turbulent spectra and PFSS models.

[44] M. D. Altschuler, Sol. Phys. 1, 377 (1967).

[45] K. H. Schatten and J. M. Wilcox, J. Geophys. Res. 72, 5185 (1967).

[46] Y. M. Wang and J. Sheeley, N. R., Astrophys. J. 392, 310 (1992).

[47] S. T. Badman, S. D. Bale, J. C. Martínez Oliveros, O. Panasenco, M. Velli, D. Stansby, J. C. Buitrago-Casas, V. Réville, J. W. Bonnell, A. W. Case, T. Dudok de Wit, K. Goetz, P. R. Harvey, J. C. Kasper, K. E. Korreck, D. E. Larson, R. Livi, R. J. MacDowall, D. M. Malaspina, M. Pulupa, M. L. Stevens, and P. L. Whittlesey, Astrophys. J. Suppl. Ser. 246, 23 (2020).

[48] J. T. Nolte and E. C. Roelof, Sol. Phys. 33, 241 (1973).

[49] A. R. Macneil, M. J. Owens, A. J. Finley, and S. P. Matt, Mon. Not. R. Astron. Soc. 509, 2390 (2021).

[50] S. K. Antiochos, Z. Mikić, V. S. Titov, R. Lionello, and J. A. Linker, Astrophys. J. 731, 112 (2011).

[51] R. Chhiber, A. V. Usmanov, W. H. Matthaeus, and M. L. Goldstein, Astrophys. J. Suppl. Ser. 241, 11 (2019).

[52] D. B. Wexler, M. L. Stevens, A. W. Case, and P. Song, Astrophys. J. Lett. 919, L33 (2021).

[53] B. D. G. Chandran, J. Plasma Phys. 84, 905840106 (2018).

[54] J. C. Kasper, M. L. Stevens, A. W. Case, and K. E.Korreck, PSP Solar Wind Electrons Alphas and Protons (SWEAP) SPC Ion Charge Flux Distributions, Electrical Current versus Time and Energy-per-Charge, Level 2 (L2), 0.2185 s Data, [Data set], 2020 data, NASA Space Physics Data Facility, 10.48322/wpk2-yq48.

[55] J. C. Kasper, M. L. Stevens, A. W. Case, and K. E.Korreck, PSP Solar Wind Electrons Alphas and Protons (SWEAP) SPC Ion Number Density, Velocity, and Thermal Speed Momemts and Fits, Level 3 (L3), 0.2185 s Data, [Data set], 2020 data, NASA Space Physics Data Facility, 10.48322/ 49we-tr31.

[56] R. Livi, D. E. Larson, and A. Rahmati, PSP Solar Wind Electrons Alphas and Protons (SWEAP) SPAN-A
Proton Distribution Function, Partial Moments, Instrument Frame, Level 3 (L3), 7 s Data, [Data set], 2020 data, NASA Space Physics Data Facility, 10.48322/ypyhs325.

[57] P. Whittlesey, D. E. Larson, and A. Rahmati, PSP Solar Wind Electrons Alphas and Protons (SWEAP) SPE Electron Pitch Angle Distribution, Level 3 (L3), 13.981 s Data, [Data set], 2020 data, NASA Space Physics Data Facility, 10.48322/8ync-7p95.

[58] P. Whittlesey, D. E. Larson, and A. Rahmati, PSP Solar Wind Electrons Alphas and Protons (SWEAP) SPAN-B Electron Pitch Angle Distributions, Level 3 (L3), $13.981 \mathrm{~s}$ Data, [Data set], 2020 data, NASA Space Physics Data Facility, 10.48322/ccen-5a96.

[59] S. D. Bale, R. J. MacDowal, A. Koval, M. Pulupa, T. Quinn, P. Schroeder, PSP FIELDS Fluxgate Magnetometer (MAG) Magnetic Field Vectors, Radial-Tangential-Normal, RTN, Coordinates, 4 samples/cycle, Level 2 (L2), 3.413 ms Data, [Data set], 2020 data, NASA Space Physics Data Facility, 10.48322/wqcs-a534.

[60] S. D. Bale, R. J. MacDowal, A. Koval, M. Pulupa, T. Quinn, P. Schroeder, PSP FIELDS Fluxgate Magnetometer (MAG) Magnetic Field Vectors, Radial-Tangential-Normal, RTN, Coordinates, Full Resolution, Level 2 (L2), 3.413 ms Data, [Data set], 2020 data, NASA Space Physics Data Facility, 10.48322/0yy0-ba92.

[61] S. D. Bale, M. Pulupa, P. R. Harvey, T. Quinn, and P. Schroeder, Parker Solar Probe, FIELDS Radio Frequency Spectrometer, RFS, Low Frequency Receiver, LFR, Spectra, Level 2 (L2), $7 \mathrm{~s}$ and $56 \mathrm{~s}$ Data, [Data set], 2020 data, NASA Space Physics Data Facility, 10.48322/re4h-tk40.

[62] SWEAP, http://sweap.cfa.harvard.edu/.

[63] D. Stansby, A. Yeates, and S. Badman, J. Open Source Software 5, 2732 (2020).

Correction: The first sentence of the caption to Fig. 2 contained an error and has been fixed. 